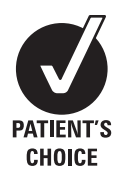

Department of Geriatric Medicine, Merlin Park University Hospital, Galway, Ireland

Correspondence to Dr S O'Keeffe, Department of Geriatric Medicine, Unit 4 Merlin Park University Hospital, Galway, Ireland: sokanc@iolfree.ie

Received 12 April 2010 Revised 30 July 2010 Accepted 5 August 2010 Published Online First 18 September 2010

\title{
Orientation to time as a guide to the presence and severity of cognitive impairment in older hospital patients
}

\author{
Emma O'Keeffe, Osman Mukhtar, Shaun T O'Keeffe
}

ABSTRACT

Background Testing of orientation to time is an important part of mental status examination. The validity of errors in different aspects of temporal orientation was examined in older hospital patients as a guide to the presence of dementia or delirium and as a measure of the severity of dementia, as defined by the Global Deterioration Scale.

Methods Inpatients and outpatients attending an acute hospital underwent independent assessments by two doctors on the same day to determine orientation to time and cognitive status. Optimum cut-offs for error scores on the different aspects of temporal orientation were calculated to maximise the sum of sensitivity and specificity for detection of dementia or delirium.

Results Of the 262 patients assessed, 62 (23.7\%) had dementia or delirium. The best cut-offs for detection of these disorders were: any error in identifying the year, month, day of the month or day of the week; and an error of more than $1 \mathrm{~h}$ in identifying the time of day. Failure to identify the year correctly was the most valuable single sign of dementia or delirium (sensitivity $86 \%$ and specificity $94 \%$ ); failure to identify either year or month correctly was $95 \%$ sensitive and $86.5 \%$ specific for the detection of cognitive impairment. Severity of temporal disorientation, measured using a number of approaches, was strongly associated with severity of dementia.

Conclusion Disorientation to time is a useful guide to the presence and severity of dementia or delirium in older hospital patients. Failure to identify the year or month correctly is a sufficiently sensitive and specific indicator of dementia or delirium to warrant more detailed cognitive assessment in this population.

Disorientation to time is a very common feature of delirium and dementia, and assessment of temporal orientation is a well established and routine part of mental status testing. ${ }^{1}$ There is no difficulty in recognising abnormality in patients with gross temporal disorientation. However, clinicians are sometimes uncertain about the significance of more minor orientation errors, such as misstating the day of the month or the week by a day or two, as a guide to possible delirium or dementia in older people. $^{2}$

Studies in young and middle aged subjects show that it is rare for a normal individual to misidentify the year or the month. ${ }^{3}$ The most common error is to misidentify the day of the month. However, less than $5 \%$ misidentify the day of the month by more than 3 days. Benton et al reported that there was 'no evidence of a decline' in temporal orientation with age in a study of 162 community volunteers aged between 65 and 84 years (1981). ${ }^{4}$ Nevertheless, the frequency of temporal disorientation in their study was $1 \%$ in 90 patients aged $65-74$ years and $6 \%$ in 72 patients aged $75-84$ years. Brotchie et al used a different questionnaire to study temporal orientation in 235 hospital visitors aged 50-84 years. ${ }^{5}$ They noted that less than $2 \%$ misstated the year or month but that errors in identifying the day of the month became more common with increased age. Studies in patients with possible dementia suggest that temporal orientation is a useful screening test for this disorder. ${ }^{6}$ Furthermore, studies in head injury, alcohol withdrawal ${ }^{9}$ and dementia ${ }^{10}$ indicate that the magnitude of errors on orientation tests is predictive of the severity of impairment on more detailed neuropsychological testing.

In the only previous study, to our knowledge, to examine the significance of errors in different aspects of temporal orientation in older hospital patients, all errors were sensitive to cognitive impairment but only failure to identify the year, month or day of the week correctly had a specificity greater than $70 \%$ for identification of dementia or delirium. ${ }^{11}$ However, the fact that patients were studied just after admission may have exaggerated the specificity of abnormalities since it seems possible that orientation to time might worsen, even in normal subjects, with prolonged admission. Also, the fact that the same examiner assessed cognitive status and temporal orientation was a possible source of bias.

In this study, we examined the validity of errors in different aspects of temporal orientation as a guide to the presence or absence of cognitive impairment (dementia, delirium or both) in older hospital patients. We also examined whether the severity of temporal disorientation might provide a useful measure of the severity of dementia in this population.

\section{METHODS \\ Patients and setting}

Subjects recruited for this study were a convenience sample of patients aged 65 years or more present during a series of cross sectional surveys of inpatients on the general medical wards of a university teaching hospital and among those attending the geriatric outpatient clinics during September to November 2009. Subjects with major communication difficulties (unable to speak English, severe deafness or such severe cognitive impairment that communication was impossible), those considered by their doctors or nurses to be too sick to be 
questioned and those who were unwilling to participate were excluded. The study was approved by the local clinical research ethics committee.

\section{Assessment}

Subjects were assessed separately by two different doctors on the same day. The first doctor explained the study and sought written consent or witnessed verbal assent to proceed. This doctor then examined temporal orientation by asking for the year, the month, the date (day of the month), the day of the week and, making sure the patient could not look at a watch or clock, the time of day. Answers were recorded in the patients' own words. Patients who said they could not answer or were unsure about any item were encouraged to provide their best estimate. Demographic information, including age, sex, date of birth and length of hospital stay, were obtained from the medical notes.

Subsequently, all patients were interviewed independently by an experienced clinician who determined whether they had cognitive impairment (delirium or dementia or both). Details of prior cognitive function were sought from all available sources, including carers, health care professionals and medical and nursing notes. Delirium was defined according to the Confusion Assessment Method in the presence of: (1) acute onset and fluctuating course; and (2) difficulty focusing or maintaining attention; and either (3) disorganised thinking or (4) altered level of consciousness. ${ }^{12}$ Dementia was staged according to the Global Deterioration Scale, a well established and validated clinical rating system comprising detailed descriptions of seven stages ranging from normal cognition to very severe dementia. ${ }^{13} 14$ For the purpose of analysis in this study, these seven stages were reduced to five: absent (stages 1 and 2), questionable (stage 3), mild (stage 4), moderate (stage 5) or severe (stages 6 and 7).

\section{Sample size calculation}

Sample size calculation was based on the approach recommended by Guyatt et al for diagnostic test studies. ${ }^{15}$ We assumed that at least $20 \%$ of subjects would have cognitive impairment and that the best test would have a sensitivity of $85 \%$ for detection of cognitive impairment. ${ }^{11}$ The required sample size to measure this sensitivity with a $95 \%$ confidence interval of no more than $10 \%$ was 246 .

\section{Analyses}

All analyses were performed using SPSS V.15.0 for windows. Data were inspected to determine the frequency and nature of errors, including non-responses, in those without cognitive impairment. The hypothesis that orientation errors became more common with increased length of hospital stay was assessed using non-parametric tests.

This study was concerned primarily with two potential uses of temporal orientation: (1) predicting cognitive impairment (delirium or dementia or both) from temporal disorientation; and (2) using severity of temporal disorientation as a guide to the severity of dementia.

\section{Predicting cognitive impairment from temporal disorientation}

Error scores for the different components of temporal orientation (year, date, day, month and time) were examined in patients with and without cognitive impairment. A single error point was given for each 30 min, day of week, day of month, month or year removed from the correct answer. It was considered likely that failure to provide an answer for any aspect of temporal orientation would be most likely in those with cognitive impairment; hence, non-responses received 2 error points. The area under receiver operating characteristic (ROC) curves relating the different aspects of temporal orientation to a diagnosis of cognitive impairment were examined; this measure can take values between 0 and 1 , where 1 is a perfect screening test and 0.5 is a test equal to chance. Examination of the ROC curves was used to select the cut-off points in error scores for each aspect that maximised the sum of sensitivity and specificity. Sensitivity, specificity and positive and negative likelihood ratios (with 95\% CI) were calculated for each cut-off.

Stepwise logistic regression was used to determine the optimal combination of orientation items with the intention of deriving a simple model for predicting the presence or absence of cognitive impairment from temporal orientation items alone. The Nagelkerke R square, which ranges between 0 (no association) and 1 (perfect association), was used to provide a guide to the power (effect size) of the model ${ }^{16}$; this can be regarded as an approximation of the ordinary least squares $\mathrm{R}$ square in linear regression which measures the proportion of variance in the outcome explained by the regression model.

\section{Severity of temporal disorientation as a measure of severity of cognitive impairment}

The relationships between dementia stage, as a measure of severity of cognitive impairment, and errors in different aspects of orientation were examined using the Jonckheere-Terpstra test, a non-parametric equivalent of $\chi^{2}$ for trend. ${ }^{16} 17$ The same test was used to assess the relationship between dementia stage and simple additive and weighted approaches to measuring temporal orientation error scores. In the additive approach, a single error point was given for any error in year, month, date, day of the week or time, and these were summed to give an overall score from 0 to 5 . In the weighted approach, we calculated the Temporal Orientation Scale (TOS) described by Benton et al with, as before, a double score being given for non-responses in any domain (box 1). ${ }^{18}$ Subjects with delirium, with or without coexisting dementia, were excluded from all of these analyses.

\section{RESULTS}

Of the 262 patients included in the study, 169 were inpatients and 93 were outpatients. There were 151women and 111 men;

Box 1 Scoring system for Benton's Temporal Orientation Scale

Time of day: 1 point for each 30 min removed from correct time (maximum 5 points).

- Day of week: 1 point for each day removed from correct day (maximum 3 points).

- Day of month: 1 point for each day removed from correct day (maximum 15 points).

- Month: 5 points for each month removed from correct month, but if date is within 15 days of correct date, no additional points are scored for the incorrect month; for example, 28 July instead of 2 August gets 5 points.

- Year: 10 error points for each year removed from correct year (maximum 60 points) but, if date is within 15 days of correct date, no additional points are scored for the incorrect year; for example, December 282008 for 2 January 2009 gets 5 error points.

- The total number of points equals the patient's score on the test. 
median (range) age was $76(65-101)$ years. Overall, $62(23.7 \%)$ patients (15 (16.1\%) outpatients and $47(27.8 \%)$ inpatients) had cognitive impairment: 15 (all inpatients) had delirium (11 of whom also had dementia) and 47 had dementia (mild in 26, moderate in 12 , severe in 9 ); an additional 18 subjects $(6.9 \%$ of the study population) had questionable dementia.

The frequency of non-responses for different aspects of temporal orientation among the 262 patients were $25(9.5 \%)$ for date, $12(4.6 \%)$ for time of day, 10 (3.8\%) for day of the week, eight $(3.1 \%)$ for month and seven $(2.7 \%)$ for year. Inspection of the data showed that non-responses were more common with increasing cognitive impairment. Among the 200 patients without cognitive impairment, the frequency of non-responses were eight $(4.0 \%)$ for date, four $(2.0 \%)$ for time of day, three $(1.5 \%)$ for day of the week, one $(0.5 \%)$ for month and $0 \%$ for year.

In the 200 patients without cognitive impairment, the following was the frequency of error (or failure to respond): date in $123(61.5 \%)$; time (30 min or more) in 98 (49\%); day of the week in $36(18 \%)$; month in $28(13 \%)$; and year in $12(6 \%)$. Most errors were relatively minor: thus, again, including failure to respond among the errors, 76 (38\%) had the date wrong by 3 days or more, $61(30.5 \%)$ had the time wrong by an hour or more, $12(6 \%)$ had the day of the week wrong by more than 1 day, four ( $2 \%$ ) had the month wrong by more than 1 month and five $(2.5 \%)$ had the year wrong by more than 1 year. Also, of the 28 people who made an error in identifying the month, nine made their error when the date of testing was 1 or 2 days either side of the start of the month.

Visual inspection and analysis of the data in those without cognitive impairment showed a significant relationship between the presence and the magnitude of errors in identifying the date and the day of the week and increased length of hospital stay (LOS). For date, median LOS was 0 (range $0-78$ ) for those with no error and 6 (0-67) for those with an error, p (Mann-Whitney $U$ test) $<0.0001$; there was a positive correlation (Spearman's rho 0.28, $\mathrm{p}<0.0001$ ) between the size of the error and LOS (outpatients being given an LOS of 0 ). For day of the week, median LOS was 3 (range 0-169) for those with no error and 7 (0-67) for those with an error, $p=0.002$; Spearman's rho was $0.21(p=0.003)$. For year, median LOS was 3 (range $0-133)$ for those with no error and $6(0-169)$ for those with an error, $p=0.08$. For month, median LOS was 4 (range $0-78$ ) for those with no error and $4(0-169)$ for those with an error, $p=0.65$. For time of day, median LOS was 3 (range $0-78$ ) for those with no error and 4 (0-169) for those with an error, $\mathrm{p}=0.1$.

\section{Predicting cognitive impairment from temporal disorientation}

The best cut-offs for detection of cognitive impairment were: any error in identifying the year, month, day of the week or date, and an error of $1 \mathrm{~h}$ or more in identifying the time of day. The test characteristics associated with these cut-offs are shown in table 1 . Areas under the ROC curve $(95 \% \mathrm{CI}$, significance level) for detection of cognitive impairment were: 0.92 (0.86 to 0.97, $\mathrm{p}<0.0001)$ for error in year, $0.80(0.72$ to $0.87, \mathrm{p}<0.0001)$ for error in month, $0.73(0.67$ to $0.80, \mathrm{p}<0.0001)$ for error in time of day, $0.70(0.62$ to $0.78, p<0.0001)$ for error in day of the week and 0.61 ( 0.54 to $0.69, p=0.006)$ for error in date. (If the definition of error in identifying month was changed to exclude errors within 2 days of a change in month, sensitivity of an error decreased to $0.66(41 / 62)$ while specificity increased to $0.95(190 /$ $200)$; the area under the ROC curve was almost identical at 0.79 $(0.72-0.87))$.

Of the 15 subjects with delirium, all misidentified (or failed to respond to) the year and the day of the month, 14 (93.3\%) did not know the time and $12(80.0 \%)$ did not know the month or day of the week.

In a backward stepwise logistic regression, not knowing the year (odds ratio (OR) 37.2 (95\% CI (CI) 13.1 to 101.3), not knowing the month (OR 3.85 (1.43 to 10.38) and error of $1 \mathrm{~h}$ or more in time of day (OR 2.76 (1.0 to 7.46) were independent predictors of cognitive impairment (Nagelkerke $R$ square 0.70 ). On examination of the different possible combinations of these three factors, the highest sum of sensitivity and specificity for the detection of cognitive impairment was provided by the presence of either error in year or month (95\% sensitivity (59/ $62)$ and $86.5 \%$ specificity $(173 / 200))$.

\section{Severity of temporal disorientation as a measure of severity of cognitive impairment}

The relationship between dementia stage and the magnitude of orientation errors are shown in table 2; results for delirious patients are included for comparison. Standardised JonckheereTerpstra statistics showed that scores on all measures, except error in identifying the date, increased significantly with increasing severity of dementia. Effect size provides a useful metric for comparing the relative magnitude of the differences between the groups with each measure of severity: as a rough guide, a large effect size is one that is equal to or greater than 0.80 , a medium effect is one that is equal to or greater than 0.50 but less than 0.80 , and a small effect is one that is equal to or greater than 0.20 but less than 0.50 . These results suggest that while the TOS is slightly more responsive than the additive test for assessing severity of temporal orientation, assessing the magnitude of error in year on its own was an even better measure in this population. The boxplot relating error in year to dementia stage is shown in figure 1.

\section{DISCUSSION}

Our results confirm the value of testing temporal orientation in older hospital patients and establish useful cut-offs for identifying dementia or delirium in this group. Failure to identify the year correctly was the most valuable single sign of cognitive impairment, combining high sensitivity and specificity, while the combination of either error in identifying year or month provided the best combination of sensitivity and specificity.

Table 1 Sensitivity, specificity and likelihood ratios (with 95\% Cls) of temporal orientation tests for the diagnosis of cognitive impairment

\begin{tabular}{|c|c|c|c|c|}
\hline Test & Sensitivity & Specificity & +LR & - LR \\
\hline Year (any error) & $0.86(0.78$ to 0.91$)$ & $0.94(0.92$ to 0.96$)$ & $14.25(9.15$ to 21.33$)$ & $0.15(0.09$ to 0.25$)$ \\
\hline Month (any error) & $0.69(0.60$ to 0.78$)$ & $0.86(0.83$ to 0.89$)$ & 4.95 (3.50 to 6.82$)$ & $0.36(0.25$ to 0.49$)$ \\
\hline Date (any error) & 0.95 (0.88 to 0.98$)$ & $0.38(0.36$ to 0.39$)$ & $1.55(1.36$ to 1.27$)$ & $0.13(0.04$ to 0.35$)$ \\
\hline Day of week (any error) & $0.58(0.48$ to 0.68$)$ & $0.82(0.79$ to 0.85$)$ & $3.23(2.26$ to 4.48$)$ & $0.51(0.38$ to 0.66$)$ \\
\hline Time of day ( $\geq 1 \mathrm{~h}$ error) & $0.77(0.67$ to 0.86$)$ & $0.70(0.66$ to 0.72$)$ & $2.54(2.00$ to 3.05$)$ & 0.330 .20 to 0.50 ) \\
\hline
\end{tabular}

LR, likelihood ratio. 
Table 2 Relationship of different severity scores to dementia stage

\begin{tabular}{|c|c|c|c|c|c|c|c|}
\hline & \multicolumn{7}{|c|}{ Measure of severity (median (range)) } \\
\hline & Year & Month & Day & Date & Time & Additive & TOS \\
\hline \multicolumn{8}{|l|}{ Dementia stage } \\
\hline Questionable $(n=18)$ & $0(0-9)$ & $0(0-2)$ & $0(0-2)$ & $1(0-7)$ & $0(0-5)$ & $2(0-4)$ & $6(0-70)$ \\
\hline Mild $(n=26)$ & $4(0-32)$ & $1(0-2)$ & $0(0-3)$ & $1(0-12)$ & $2(0-4)$ & $3(1-5)$ & $43(1-80)$ \\
\hline Moderate $(n=12)$ & $8(0-50)$ & $1(0-3)$ & $1(0-3)$ & $2(1-14)$ & $2(1-6)$ & $5(4-5)$ & $68(29-98)$ \\
\hline J-T statistic & 12.2 & 8.9 & 1.9 & 6.7 & 5.3 & 8.3 & 9.9 \\
\hline $\mathrm{p}$ Value & $<0.0001$ & $<0.0001$ & 0.06 & $<0.0001$ & $<0.0001$ & $<0.0001$ & $<0.0001$ \\
\hline Effect size & 0.78 & 0.57 & 0.12 & 0.43 & 0.34 & 0.53 & 0.63 \\
\hline
\end{tabular}

J-T statistic, standardised Jonckheere-Terpstra statistic; TOS, Temporal Orientation Scale.

Molloy et al recommended in their standardised version of the Mini-Mental State Examination (MMSE) that the responses to the month item should be accepted as correct if, on the first day of a new month or the last day of the previous month, people say either of these months. ${ }^{19}$ In the current study, many errors in identifying the month in cognitively normal people occurred within 2 days either side of the new month; however, improved specificity with using this diagnosis of error in identifying the month was more than offset by a reduction in sensitivity to cognitive impairment.

Even when using optimal cut-offs, assessment of the day of the month, the day of the week and the time of day were less helpful tests for identifying cognitive impairment in this study although, because inability to identify the day of the month was so common in those with cognitive impairment, the absence of such an error might be useful in effectively ruling out a significant cognitive problem. There was a strong relationship between the duration of hospital stay and both the presence and the magnitude of errors in identifying date of the month and day of the week. This is not surprising given the relative absence of usual social and environmental cues during prolonged hospitalisation.

The assumption that errors in knowing the year or the month are more likely to be significant than mistakes such as misstating

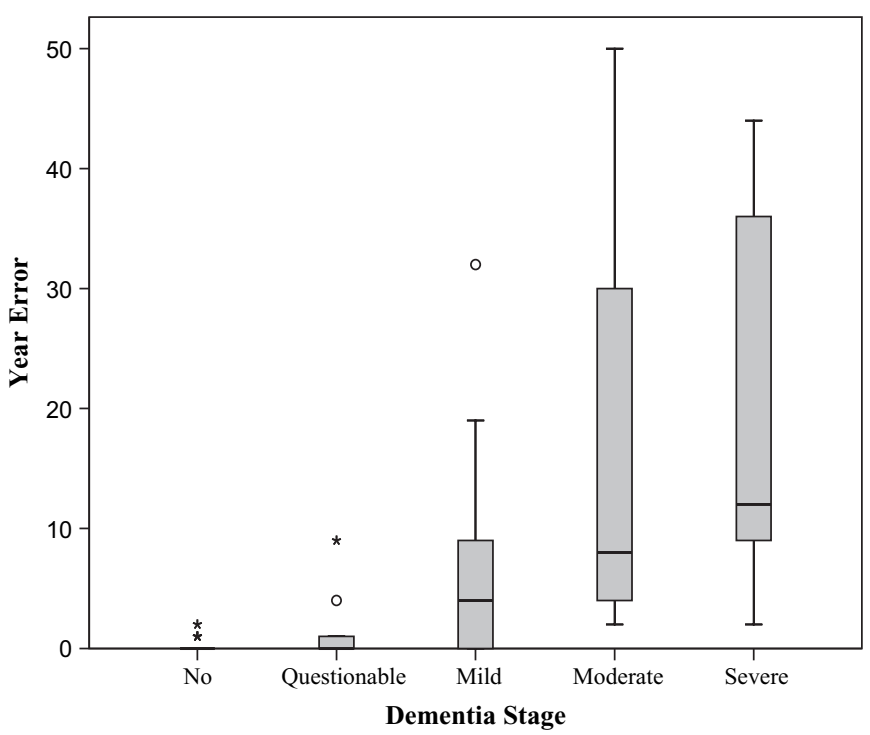

Figure 1 Boxplot relating error in year to dementia stage. A minor outlier (denoted by a circle) is an observation 1.5 times the interquartile range outside the central box. A major outlier (denoted by ${ }^{*}$ ) is an observation 3.0 times the interquartile range outside the central box. the day of the month by a day or two is reflected in the TOS which has differently weighted scores for different elements of orientation, with errors in month and particularly year attracting higher error scores than errors in day or date. ${ }^{18}$ However, in the MMSE, ${ }^{20}$ questions regarding the season, day, month, year or date are scored equally as correct or incorrect, and a similar approach is taken in other brief cognitive tests. ${ }^{21} 22$ Both approaches to measuring severity of temporal disorientation have been moderately predictive of performance on more detailed cognitive testing in a variety of patient groups. ${ }^{9} 1023$

In the current study, both the additive scores and the TOS increased significantly with increasing severity of dementia. However, the greater effect size of the TOS supports the value of using a weighted scoring system. Further support for this approach comes from the finding that higher weighting of year error scores in the Modified Mini-Mental State Examination $(3 \mathrm{MS}),{ }^{24}$ a revision of the MMSE, results in increased discrimination between normal cognitive function and Alzheimer's disease patients. ${ }^{25}$

Although the TOS remains the best standardised psychometric test of temporal orientation, the complexity of the scoring system is a disadvantage. The current study also suggests that the capping of year error scores when a 6 year error is reached is too restrictive; in our older hospital population, errors of some decades were not uncommon and occurred mainly in those with moderate to severe dementia. Indeed, our results suggest that reliance on year error scores alone might be a simple alternative in an older population.

Although the number of patients with delirium in the current study was small, orientation errors were very sensitive to delirium, and the severity of disorientation in delirious patients was comparable with that seen in those with severe dementia. The relationship between the severity of temporal disorientation and of dementia raises the question of whether severity of disorientation might also be a simple marker of the severity of delirium and, as Varney and Shephard suggested, ${ }^{9}$ whether serial assessment of temporal orientation might be useful in monitoring change in cognitive status in patients with delirium. Studies in head injury patients support the value of serial assessment of orientation ${ }^{8} 26$ but this requires further assessment in delirium.

The independent assessment of temporal orientation and of cognitive status on the same day in all subjects is an advantage of this study. The use of a convenience sample of patients is a major disadvantage of the study since this approach gives rise to concern about sampling bias and limits the generalisability of the study. Furthermore, we did not assess the educational status of our patients although there is evidence that errors are more 
common in those with less education. ${ }^{27}$ The evidence in this study for the superiority of the weighted approach to assessing severity of disorientation is indirect, and numbers were inadequate to examine the relative ability of different scoring systems to distinguish different stages of dementia.

Recognition of dementia and, more importantly, delirium in hospital patients by medical and nursing staff is notoriously poor. ${ }^{28}$ Orientation is often the only aspect of cognition assessed by doctors and nurses, usually in the form of a cursory comment that the patient is 'oriented to time, place and person'-or the contrary-based on their own interpretation of what errors might be acceptable. ${ }^{28-30}$ Testing temporal orientation is not an adequate substitute for proper mental status testing or for knowledge of cognitive problems. However, if cognitive testing is to be restricted to a single domain, orientation to time is one of the most efficient measures for discriminating between those with and without dementia and in predicting cognitive decline with time. ${ }^{6} 3132$ Furthermore, the reliance on assessment of orientation has proven relatively resistant to educational interventions. ${ }^{33}$ Our results suggest that temporal orientation, properly recorded and interpreted, may provide a useful screening test for dementia or delirium in older hospital patients. In particular, failure to identify the year or month correctly is a sufficiently sensitive and specific indicator of dementia or delirium in older hospital patients to warrant specialist referral for more detailed assessment in this population.

Competing interests None.

Ethics approval This study was conducted with the approval of the Clinical Research Ethics Committee, Galway University Hospitals.

Provenance and peer review Not commissioned; externally peer reviewed.

\section{REFERENCES}

1. Berrios GE. Orientation failures in medicine and psychiatry: discussion paper. J $R$ Soc Med 1983;76:379-85.

2. Molloy DW, Standish TI. A guide to the standardized Mini-Mental State Examination. Int Psychogeriatr 1997;9(Suppl 1):87-94.

3. Benton AL, Sivan AB, Hamsher KD, et al. Temporal orientation. contributions to neuropsychological assessment. 2nd Edn. New york: Oxford University Press, 1994:3-11.

4. Benton AL, Eslinger PJ, Damasio AR. Normative observations on neuropsychological test performances in old age. J Clin Neuropsychol 1981;3:33-42.

5. Brotchie J, Brennan J, Wyke MA. Temporal orientation in the pre-senium and old age. Br J Psychiatry 1985;147:692-5.

6. Tractenberg RE, Weinstein M, Weiner MF, et al. Benchmarking a test of temporal orientation with data from American and Taiwanese persons with Alzheimer's disease and American normal elderly. Neuroepidemiology 2005;24:110-16.

7. Fayers PM, Hjermstad MJ, Ranhoff AH, et al. Which Mini-Mental State Exam items can be used to screen for delirium and cognitive impairment? J Pain Symptom Manage 2005;30:41-50.
8. High WM Jr, Levin HS, Gary HE Jr. Recovery of orientation following closed-head injury. J Clin Exp Neuropsychol 1990;12:703-14.

9. Varney NR, Shepherd JS. Predicting short-term memory on the basis of temporal orientation. Neuropsychology 1991;5:13-16.

10. Ryan JJ, Glass LA, Bartels JM, et al. Predicting neuropsychological test performance on the basis of temporal orientation. Neuropsychol Dev Cogn B Aging Neuropsychol Cogn 2009;16:330-7.

11. O'Keeffe ST, Crowe M, Gustau B, et al. Interpreting errors in temporal orientation in older hospital patients. J Clin Geropsychol 2001;7:47-52.

12. Inouye SK, van Dyck CH, Alessi CA, et al. Clarifying confusion: the confusion assessment method. A new method for detection of delirium. Ann Intern Med 1990;113:941-8.

13. Reisberg B, Ferris SH, de Leon MJ, et al. The Global Deterioration Scale for assessment of primary degenerative dementia. Am J Psychiatry 1982;139:1136-9.

14. Overall JE, Scott J, Rhoades HM, et al. Empirical scaling of the stages of cognitive decline in senile dementia. J Geriatr Psychiatry Neurol 1990;3:212-20.

15. Guyatt G, Sackett DBH. Evaluating diagnostic tests. In: Hynes DS, Guyatt GH Tugwell P, eds. Clinical epidemiology. How to do clinical practice research, 3rd Edn. Philadelphia: Williams and Wilkins, 2006:273-322.

16. Field A. Discovering statistics using SPSS, 3rd Edn. London: Sage, 2009:264-319

17. Mehotcheva TH. The Kruskal-Wallis Test. http://www.let.rug.nl/ nerbonne/ teach/rema-stats-meth-seminar/presentations/Mehotcheva-2008-Kruskal-Wallis.pdf (accessed 20 Jul 2010).

18. Benton AL, Vanallen MW, Fogel ML. Temporal orientation in cerebral disease. J Nerv Ment Dis 1964:139:110-19.

19. Molloy DW, Alemayehu E, Roberts R. Reliability of a Standardized Mini-Mental State Examination compared with the traditional Mini-Mental State Examination. Am J Psychiatry 1991:148:102-5.

20. Tombaugh TN, Mclntyre NJ. The Mini-Mental State Examination: a comprehensive review. J Am Geriatr Soc 1992;40:922-35.

21. Jitapunkul S, Pillay I, Ebrahim S. The abbreviated mental test: its use and validity. Age Ageing 1991;20:332-6.

22. Nasreddine ZS, Phillips NA, Bedirian V, et al. The Montreal Cognitive Assessment, MoCA: a brief screening tool for mild cognitive impairment. J Am Geriatr Soc 2005:53:695-9.

23. Desmond DW, Tatemichi TK, Figueroa $\mathrm{M}$, et al. Disorientation following stroke: frequency, course, and clinical correlates. J Neurol 1994;241:585-91.

24. Teng EL, Chui HC. The Modified Mini-Mental State (3MS) examination. J Clin Psychiatry 1987:48:314-18.

25. Tombaugh TN. Test-retest reliable coefficients and 5-year change scores for the MMSE and 3MS. Arch Clin Neuropsychol 2005;20:485-503.

26. Bode RK, Heinemann AW, Semik P. Measurement properties of the Galveston Orientation and Amnesia Test (GOAT) and improvement patterns during inpatient rehabilitation. J Head Trauma Rehabil 2000;15:637-55

27. Natelson BH, Haupt EJ, Fleischer EJ, et al. Temporal orientation and education. A direct relationship in normal people. Arch Neurol 1979;36:444-6.

28. Inouye SK, Foreman MD, Mion LC, et al. Nurses' recognition of delirium and its symptoms: comparison of nurse and researcher ratings. Arch Intern Med 2001;161:2467-73

29. Rockwood K. Educational interventions in delirium. Dement Geriatr Cogn Disord 1999;10:426-9.

30. Dellasega C, Cutezo E. Strategies used by home health nurses to assess the menta status of homebound elders. J Community Health Nurs 1994;11:129-38.

31. Rockwood K, Black S, Bedard MA, et al. Specific symptomatic changes following donepezil treatment of Alzheimer's disease: a multi-centre, primary care, open-labe study. Int J Geriatr Psychiatry 2007;22:312-19.

32. Lou MF, Dai YT, Huang GS, et al. Postoperative cognitive changes among older Taiwanese patients. J Clin Nurs 2003;12:579-88.

33. Rockwood K, Cosway S, Stolee P, et al. Increasing the recognition of delirium in elderly patients. J Am Geriatr Soc 1994;42:252-6. 